\title{
Seven pulsars in binary systems above the spin-up line
}

\author{
Y. Y. Pan and N. Wang \\ Xinjiang Astronomical Observatories, Chinese Academy of Sciences, Xinjiang 830011, China \\ Email: panyuanyue@xao.ac.cn
}

\begin{abstract}
Using data from the ATNF pulsar catalogue, 186 binary pulsars are shown in the magnetic field versus spin period (B-P) diagram, and their relationship to the spin-up line is investigated. Generally speaking, pulsars in binary systems should be below the spin-up line when they get enough accretion mass from their companions. It is found that there are seven binary pulsars above the spin-up line. Based on the parameters of these seven binary systems, we describe possible reasons why they are above the spin-up line.
\end{abstract}

Keywords. Pulsar, binary, spin-up line

\section{Introduction}

A binary pulsar system is a pulsar with a companion, often a white dwarf, neutron star or massive star. When a neutron star is formed from the supernova, it has a high magnetic field of around $10^{11-13} \mathrm{G}$ and slow spin period of around $0.1-10 \mathrm{~s}$. In a binary system, with the accretion mass of $0.1 \sim 0.2 M_{\odot}$ from the companion, a neutron star will be spun up to several milliseconds, while its magnetic field will decrease to $\sim 10^{8-9} \mathrm{G}$ (Bhattacharya \& van den Heuvel 1991;Manchester 2004; Stairs 2004; Manchester, Hobbs, Teoh \& Hobbs 2005; Wang, Zhang \& Zhao et al. 2011; Zhang, Wang \& Zhao et al. 2011). During the accretion, the flow drag the field lines asides to dilute the polar field strength (Zhang \& Kojima 2006). When a neutron star gets to its minimum spin period to which such a spin up proceeds in an Eddington-limited accretion, we can get the spin-up line (Bhattacharya \& van den Heuvel 1991): $B_{9}^{\frac{6}{7}}=\frac{P}{2.2} R_{6}^{-\frac{18}{7}} m^{\frac{8}{7}} \times 10^{3}$, where $B_{9}$ is the magnetic field in units of $10^{9} \mathrm{G}, R_{6}$ is the stellar radius in units of $10^{6} \mathrm{~cm}, \mathrm{~m}$ is in unit of solar mass. After the accretion phase finishing, the radio emission of the fast rotating neutron star can be detected as millisecond pulsar whose spin period is less than 20 milliseconds (Alpar, Cheng, Ruderman \& Shaham 1982; Tauris 2012).

\section{Evolution of pulsars in binary systems}

Up to now 186 pulsars (including 136 millisecond ones) have been found in binary systems (data from ATNF pulsar catalogue). Fig. 1 shows us their distribution in the B$\mathrm{P}$ diagram. In binary systems, pulsars will evolve below the spin-up line after accreting a sufficient amount of mass from their companions. However, there are seven binary pulsars which lie above the spin-up line, as shown in Fig. 1. We arranged them into two groups according to their companion masses: the first one with the massive companions $\left(M>4.0 M_{\odot}\right.$, No. $\left.1 \sim 4\right)$ and the second one with the degenerate stars (white dwarf or neutron star, No. $5 \sim 7$ ). The parameters of seven binary pulsars are listed in Table 1 .

The common characteristics of the first group (No. 1 to 4) are their massive and nonrecycled companions. They all have high eccentricities and long orbital periods. The accretion phase has not yet started. Due to these characteristics, it can be said that 


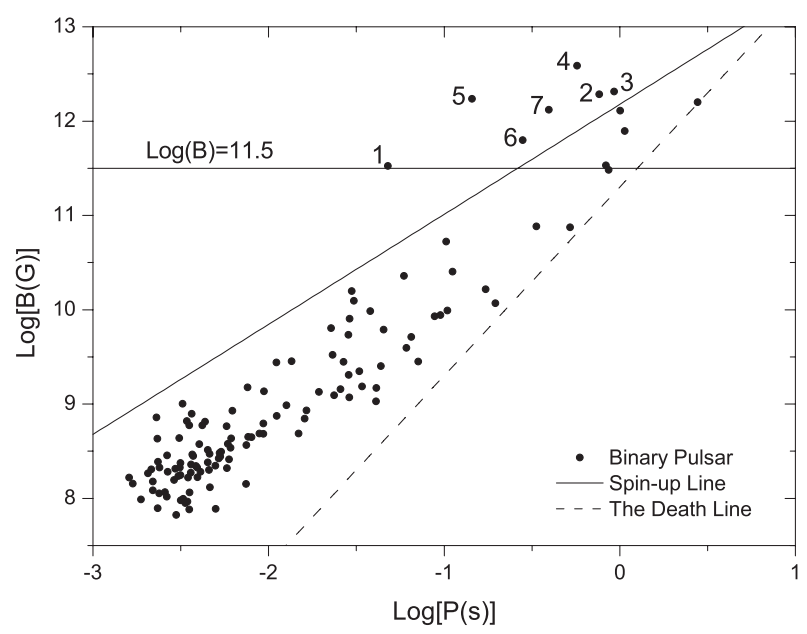

Figure 1. Magnetic field versus spin period diagram for 186 pulsars in binary system, data from ATNF pulsar catalogue. Seven pulsars are above the spin-up line which are signed by arranged number. The horizontal line is the magnetic field $B=10^{11.5} \mathrm{G}$. The solid and dash lines are the spin-up line and death line.

they are un-recycled pulsars whose companions are still in the main sequence in binary systems. Or they are on the way of evolution, where the first born pulsars are experiencing the spin-down with no accretion.

The second group (No. 5 to 7 ) includes the recycled pulsars. PSR J1906+0746 (No. 5 in the Fig. 1$)$ with mass $\left(1.25 M_{\odot}\right)$ has a neutron star companion of mass $1.37 M_{\odot}$. Comparing the mass of the two neutron stars, it is inferred that the heavier one is a recycled pulsar and the lighter one is a non-recycled pulsar. From the evolution history of double neutron stars, the heavier progenitor star explodes first to form a neutron star, and then the lighter one evolves until to its supernova explosion. During the evolution of the second star, the first formed neutron star will accrete matter, leading to its recycling. The short characteristic age of J1906+0746 ( $\tau=113000 \mathrm{yr}$ ) indicates that it is a recently formed young pulsar after the core collapse, which is the reason why its B-P position lies above the spin-up line.

B1820-11 (No. 6 in Fig. 1) possesses a slightly massive white dwarf $\left(M_{c}=0.78 M_{\odot}\right)$ as its companion. From its parameters $\left(\tau=3.22 \mathrm{Myr}, \mathrm{B}=6.29 \times 10^{11} \mathrm{G}\right.$ and $\left.P=279.829 \mathrm{~ms}\right)$, we suggest that it is a young recycled pulsar. If we reconsider its radius as $15 \mathrm{~km}$ or $20 \mathrm{~km}$ instead of $10 \mathrm{~km}$ in the spin-up line equation, then its magnetic field is about $3.0 \times 10^{11} \mathrm{G}$ and $1.6 \times 10^{11} \mathrm{G}$, respectively. Therefore, in Fig. 1, the position of B1820-11 with the new magnetic field value will lie below the spin-up line. The evolution history of this

Table 1. Information of 7 binary pulsars above the spin-up line

\begin{tabular}{lcccccccc}
\hline No. & Name & $P / m s$ & $P_{\text {orb }} / d$ & $\mathrm{e}$ & $M_{C} / M_{\odot}$ & $\tau / y r$ & $B / G$ & type \\
\hline 1 & $\mathrm{~B} 1259-63$ & 47.763 & 1236.724 & 0.8699 & 4.14 & 332000 & $3.34 \mathrm{E} 11$ & NSMS \\
2 & $\mathrm{~J} 1638-4725$ & 763.933 & 1940.9 & 0.955 & 8.08 & $2.53 \mathrm{E} 6$ & $1.93 \mathrm{E} 12$ & NSMS \\
3 & $\mathrm{~J} 0045-7319$ & 926.276 & 51.1695 & 0.8079 & 5.27 & $3.29 \mathrm{E} 6$ & $2.06 \mathrm{E} 12$ & NSMS \\
4 & $\mathrm{~J} 1740-3052$ & 570.31 & 231.0297 & 0.5789 & 15.82 & 354000 & $3.86 \mathrm{E} 12$ & NSMS \\
5 & $\mathrm{~J} 1906+0746$ & 144.072 & 0.166 & 0.0853 & 1.37 & 113000 & $1.73 \mathrm{E} 12$ & DNS \\
6 & $\mathrm{~B} 1820-11$ & 279.829 & 357.762 & 0.7946 & 0.78 & $3.22 \mathrm{E} 6$ & $6.29 \mathrm{E} 11$ & NSWD \\
7 & $\mathrm{~J} 1141-6545$ & 393.899 & 0.1977 & 0.1719 & 1.02 & $1.45 \mathrm{E} 6$ & $1.32 \mathrm{E} 12$ & NSWD \\
\hline
\end{tabular}


pulsar can be understood in this way: the initial magnetic field of neutron star can be as high as $B \sim 10^{13} \mathrm{G}$, and it can evolve from the position of long spin period to that of short spin period after the neutron star accretes about $\sim 0.001 M_{\odot}$, while one to two magnitude orders of magnetic field has been deducted.

J1141-6545 (No. 7 in Fig. 1) is a pulsar of mass $1.3 M_{\odot}$ with an $1.02 M_{\odot}$ optical white dwarf as its companion in binary system. With the short orbital period $\left(P_{\text {orbit }}=0.1977 d\right)$ and low eccentricity $(e=0.1719)$, it can be derived that this pulsar acquired the accretion mass easily and followed up the recycled process. There is about $0.001-0.01 M_{\odot}$ accretion mass added to this pulsar that can lead to its magnetic field deduce two magnitude orders from its initial values. Following the similar procedure of binary pulsar B1820-11 (No. 6), by setting the neutron star radius as large as $\mathrm{R}=20 \mathrm{~km}$, the magnetic field of J1141-6545 will be about $10^{11.6} \mathrm{G}$, which makes this source just below the spin-up line as a new born recycled pulsar. Therefore, the evolution picture of this pulsar can be depicted like this: the progenitor of J1141-6545 may be a star with the strong magnetic field $\sim 10^{13.6} \mathrm{G}$, and its field decays two magnitude orders with the accreting mass of about $0.001 M_{\odot}$.

\section{Conclusion}

In a binary system, with enough accreting matter from the companions, a pulsar should be below the spin-up line. With the distribution of 186 binary pulsars in B-P diagram, it is noticed that seven pulsars are above the spin-up line. Four of them have massive companions $\left(M>4.0 M_{\odot}\right)$ and are young pulsars which are quickly spinning down. They have not started their recycling processes. The other three binary pulsars with recycled companions have not experienced the recycled processes: one system is a double neutron system. The observed pulsar is a young one with a recycled neutron star. The other two systems include degenerate stars (NS+WD), where the pulsars can be understood as newly formed recycling pulsars at the Eddington rate; and their B-P positions can be shifted to just below the spin-up line by assuming a different neutron star radius, e.g. $R=20 \mathrm{~km}$.

This work is supported by National Basic Research Program of China (973 Program 2009CB824800), China Ministry of Science and Technology under State Key Development Program for Basic Research (2012CB821800), NSFC10773017, NSFC11173034, and Knowledge Innovation Program of CAS KJCX2- YW-T09.

\section{References}

Alpar M. A., Cheng A. F., Ruderman M. A., \& Shaham J., 1982, Nature, 300, 728

Bhattacharya D. \& van den Heuvel E. P. J. 1991, Phys. Rep., 203, 1

Camilo F., Thorsett S. E., \& Kulkarni S. R., 1994, ApJ, 421, L15

Lyne A. G., Burgay M. Kramer, M. et al., 2004, Science, 303, 1153.

Manchester R. N., 2004, Science, 304, 542

Manchester R. N., Hobbs G. B., Teoh A., \& Hobbs M., 2005, AJ, 129, 1993

Stairs I. H., 2004, Science, 304, 23

Tauris M., 2012, Science, 335, 561

van den Heuvel E. P. J., 2004, Science, 303, 20

Wang, J., Zhang, C. M., Zhao, Y. H., et al., 2011, A\&SA, 526, 88

Wijnands R. \& van der Klis M., 1998, Nature, 394, 344

Zhang C. M., Kojima Y., 2006, MNRAS, 336, 137

Zhang C. M., Wang J., Zhao Y. H. et al., 2011, A\& A, 527, 83 